\title{
Alcohol relapse and its predictors after liver transplantation for alcoholic liver disease: a systematic review and meta-analysis
}

\author{
Lancharat Chuncharunee ${ }^{1}$, Noriyo Yamashiki ${ }^{2}$, Ammarin Thakkinstian ${ }^{3}$ and Abhasnee Sobhonslidsuk ${ }^{1 *}$ (D)
}

\begin{abstract}
Background: Alcoholic liver disease (ALD) is the leading cause of liver transplantation (LT). The magnitude and risk factors of post-LT alcohol relapse are not well described. We conducted a meta-analysis to evaluate alcohol relapse rate and its predictors after LT.

Methods: Searches of MEDLINE and SCOPUS identified eligible published studies of alcohol relapse after LT published up to 31 March 2018. Alcohol relapse was defined as any alcohol consumption post-LT, and heavy alcohol relapse was defined as a relapse of alcohol consumption that was associated with a significant harm. Data for the proportion of alcohol relapse was pooled using a meta-analysis for pooling proportion. An odds ratio (OR) of the predictor of alcohol relapse was extracted and pooled using meta-analysis for the pooling risk factor. Data were analyzed using a random effect model if heterogeneity was presented; otherwise, a fixed effect model was applied. The study was registered at PROSPERO (CRD42017052659).
\end{abstract}

Results: Ninety-two studies with over 8000 cases were recruited for pooling proportion of alcohol relapse. The alcohol relapse rate and heavy alcohol relapse rate after LT during the mean follow-up time of $48.4 \pm 24.7$ months were 22\% (95\% confidence interval (Cl): 19-25\%) and 14\% (95\%Cl: 12-16\%). Psychiatric comorbidities (odds ratio (OR) 3.46, 95\%Cl: 1.87-6.39), pre-transplant abstinence of less than 6 months (OR 2.76, 95\%Cl: 2.10-3.61), unmarried status (OR 1.84, 95\%Cl: 1.39-2.43), and smoking (OR 1.72, 95\%Cl: 1.21-2.46) were associated with alcohol relapse after LT. However, we noticed publication bias of unpublished negative studies and high heterogeneity of results.

Conclusions: Post-transplant alcohol relapse occurred in about one-fifth of patients who underwent alcohol-related LT. Psychiatric comorbidities represented the strongest predictor of alcohol relapse. Psychiatric comorbidities monitoring and pre-LT alcohol abstinence for at least 6 months may decrease alcohol relapse after LT.

Keywords: Liver transplantation, Alcohol, Relapse, Recidivism, Psychiatric comorbidity

\section{Background}

Chronic and excessive alcohol consumption is a major cause of death around the world. Regular alcohol consumption can lead to steatosis, steatohepatitis, liver cirrhosis, and hepatocellular carcinoma [1-3]. Liver transplantation (LT) is an extended treatment for endstage liver diseases; alcoholic liver cirrhosis is the second most frequent cause for LT in the United States and in Europe [4]. Previous studies demonstrated that LT in

\footnotetext{
* Correspondence: abhasnee.sob@mahidol.ac.th

${ }^{1}$ Division of Gastroenterology and Hepatology, Department of Medicine, Faculty of Medicine Ramathibodi Hospital, Mahidol University, 270 Rama 6 Road, Bangkok 10400, Thailand

Full list of author information is available at the end of the article
}

ALD patients offers an equal survival rate as that in other causes of end-stage liver disease [5]. Furthermore, LT for severe alcoholic hepatitis has a favorable outcome and better survival than medical therapy, but non-surgical therapy remains the standard of care for patients with severe alcoholic hepatitis $[6,7]$.

The issues of recidivism and disease recurrence remain a concern in LT for alcoholic liver disease. Alcohol relapse negatively impacts outcomes including graft rejection and graft loss from poor medical compliance, post-transplant malignancy, cardiovascular diseases, alcoholic cirrhosis, and decreased long-term survival [8-11]. An abstinence period of at least 6 months before

(c) The Author(s). 2019 Open Access This article is distributed under the terms of the Creative Commons Attribution 4.0 International License (http://creativecommons.org/licenses/by/4.0/), which permits unrestricted use, distribution, and 
LT is a mandatory selection criterion in most liver transplant centers, but the benefit of such pre-transplant 6 month abstinence remains unclear $[8,12,13]$. Furthermore, there are subsequent reports indicating that an abstinence period of 6 months is not a significant predictive factor for recidivism [14-16]. Careful evaluation of patients with alcoholic liver disease prior to liver transplantation can identify patients with a high risk of alcohol relapse. Modifying the negative factors before LT can prevent alcohol relapse and improve post-transplant survival.

Most of these studies on alcohol recidivism after LT were done in single centers and were reported as descriptive data [16-24]. A previously published meta-analysis study of alcohol relapse after liver transplantation by Dew et al. in 2008 only included published reports on this topic up to 2004 [25]. Several predictive factors have been reported in the last decade $[8,14,15,25,26]$. Thus, we performed a systematic review and meta-analysis from the published literature with the following objectives: First, to pool prevalence of alcohol relapse after LT; second, to explore factors associated with alcohol relapse and pool their magnitude of effects in alcoholic liver disease patients with LT.

\section{Methods}

This meta-analysis was conducted by following the Preferred Reporting Items for Systematic Reviews and Metaanalysis (PRISMA) guidelines, and the review protocol was registered at PROSPERO (CRD42017052659).

\section{Search strategy}

Two investigators (L.C. and A.S.) independently conducted a search of databases via MEDLINE and SCOPUS via PubMed and Scopus search engines to identify relevant studies published up to 31 March 2018. The search terms were constructed by domains of patients, intervention/exposure, and outcome. The search strategy is outlined in Table 1. The investigators supplemented the manual reviews of article reference lists to identify studies that had not been included from the initial database search and also performed manual reviews of the relevant studies.

\section{Study selection}

Two investigators (L.C. and A.S.) determined the eligibility of each article for inclusion by screening for relevance on titles and abstracts in parallel. If an eligibility of the study was indeterminable from abstracts, then the full articles were retrieved. The kappa statistic was used to estimate the agreement between the two reviewers [27]. Discordant decisions between the two investigators were advanced to full- text review and resolved by consensus with the third investigator (A.T.).

\section{Inclusion criteria}

\section{Studies were eligible if they met all of the following criteria}

- The study was an observational design including cohort, cross-sectional, or case-control study published as an original article.

- The work studied adult patients aged over 18 years with alcoholic liver disease who underwent any type of LT.

- Reporting any of the following outcomes: alcohol relapse or alcohol recurrence.

- The study assessed association between alcohol relapse and any risk factor including seven domains as follows: demographic data, psychiatric conditions, socioeconomic status, family support, alcohol abstinence, rehabilitation program, and high-risk alcohol relapse (HRAR) scale [28].

\section{Exclusion criteria}

Studies were excluded if they met one of the below criteria:

- Non-English articles that cannot be translated.

- Studies with multi-organ transplantation.

- Insufficient data for extraction.

\section{Outcome of interest}

The outcome of interest was alcohol relapse and heavy alcohol relapse in patients who underwent LT for alcoholic liver disease. We selected papers on the occurrence of alcohol relapse based on the original authors' definition of alcohol relapse and heavy relapse and used several methods of relapse assessment such as selfreporting and collateral reporting except for biochemical testing and indirect measures. In general, alcohol relapse was defined as any alcohol consumption post-transplantation, and heavy alcohol relapse was defined as a relapse of alcohol consumption associated with significant medical or social harm [29].

\section{Data extraction}

Data obtained from each study was independently extracted by two reviewers (L.C. and A.S.) using a standardized extraction form. Study design, details of the publication, the number of subjects, and baseline characteristics of study populations were extracted including patients with alcohol relapse and predictive factors of alcohol relapse after LT. 
Table 1 Search terms and search strategy

\begin{tabular}{|c|c|c|}
\hline Domain & Search term & Search strategy \\
\hline \multirow[t]{5}{*}{ P-Patient } & - "Alcoholic hepatitis" & $\# 1$ \\
\hline & - "Alcoholic liver disease" & $\# 2$ \\
\hline & - "Alcoholic cirrhosis" & $\# 3$ \\
\hline & - "Liver transplantation" & $\# 4$ \\
\hline & - "Hepatic transplantation" & $\# 5$ \\
\hline All P & $\# 6$ & \#1 OR \#2 OR \#3 AND \#4 OR \#5 \\
\hline \multirow{29}{*}{$\begin{array}{l}\text { E-Exposure (I-intervention/C- } \\
\text { comparator) }\end{array}$} & - Gender & $\# 7$ \\
\hline & - Sex & $\# 8$ \\
\hline & $\# 9$ & \#7 OR \#8 \\
\hline & - Age & $\# 10$ \\
\hline & - "Marital status" & $\# 11$ \\
\hline & - Divorced & $\# 12$ \\
\hline & $\# 13$ & $\# 11$ OR \#12 \\
\hline & - "Socioeconomic status" & $\# 14$ \\
\hline & - Income & $\# 15$ \\
\hline & - Education & $\# 16$ \\
\hline & $\# 17$ & \#14 OR \#15 OR \#16 \\
\hline & - "Alcohol dependence" & $\# 18$ \\
\hline & - Depression & $\# 19$ \\
\hline & - "Drug use" & $\# 20$ \\
\hline & - "Substance use" & $\# 21$ \\
\hline & - "Substance abuse" & \#22 \\
\hline & - substance & $\# 23$ \\
\hline & $\# 24$ & \#20 OR \#21 OR \#22 OR \#23 \\
\hline & - Family history of alcohol & $\# 25$ \\
\hline & - Alcohol abstinence & $\# 26$ \\
\hline & - Alcohol cessation & $\# 27$ \\
\hline & - Alcohol quit & $\# 28$ \\
\hline & - Alcohol stop & $\# 29$ \\
\hline & - Alcohol sobriety & $\# 30$ \\
\hline & $\# 31$ & \#26 OR \#27 OR \#28 OR \#29 OR \#30 \\
\hline & - Rehabilitation & \#32 \\
\hline & $\begin{array}{l}\text { - High Risk Alcoholism } \\
\text { Relapse }\end{array}$ & \#33 \\
\hline & - HRAR & \#34 \\
\hline & \#35 & \#33 OR \#34 \\
\hline All E & \#36 & $\begin{array}{l}\text { \#9 OR \#10 OR \#13 OR \#17 OR \#18 OR \#19 OR \#24 OR \#25 OR \#31 OR \#32 OR } \\
\text { \#35 }\end{array}$ \\
\hline \multirow[t]{3}{*}{ O-Outcome } & - Alcohol relapse & $\# 37$ \\
\hline & - Alcohol recurrence & \#38 \\
\hline & - Recidivism & \#39 \\
\hline All O & \#40 & \#37 OR \#38 OR \#39 \\
\hline Overall & & \#6 AND \#36 AND \#40 \\
\hline
\end{tabular}




\section{Quality and risk of bias assessment}

All selected studies were independently reviewed by two investigators (L.C. and A.S.). Disagreements between the two reviewers were resolved by consensus with the third investigator (A.T.). Quality was assessed using a Newcastle-Ottawa Scale for eligible studies including selection, exposure, and comparability of studies on the basis of the design or analysis and assessment of the outcomes (Table 2).

\section{Statistical analysis}

The rate of alcohol relapse after LT was estimated along with its 95\% confidence interval (CI) for each study. The rate was then pooled across studies using a meta-analysis for pooling proportion [102]. The random effect model was applied if there was heterogeneity between studies; otherwise, a fixed-effect model was applied. An odds ratio (OR) along with 95\% CI of risk factor associated with alcohol relapse after LT was estimated for each study. Heterogeneity was assessed using the Cochrane Q test and the $\mathrm{I}^{2}$ statistic. Heterogeneity was present when the $\mathrm{Q}$ test was significant $(p<0.1)$ or $\mathrm{I}^{2} \geq 25 \%$. The sources of heterogeneity were then explored using a meta-regression if the data of the co-variables were available. Subgroup analysis by age, region of study, definition of alcohol relapse, and follow-up time was then performed accordingly. Publication bias was assessed by Egger's test and a funnel plot. If there was asymmetry suggested from either a funnel plot or Egger's test, then a contour-enhanced funnel plot was used to explore whether the asymmetry was due to publication bias or heterogeneity. All analyses were performed using STATA software version 14.1. $P$-values $<0.05$ and $<0.10$ were considered statistically significant for a two-sided test and one-sided test, respectively.

\section{Results}

\section{Search result}

A total of 291 studies were identified from PubMed and Scopus databases plus 30 additional studies from the reference lists (Fig. 1). The title and abstracts were reviewed for 321 studies; 123 duplicated studies, 1 metaanalysis, and 4 systematic reviews were removed [25, 103-106]. The remaining 193 studies were reviewed in full text excluding 101 studies. Of the remaining studies, 90 reported the proportion of alcohol relapse, and 37 studies assessed risk factors of alcohol relapse. The kappa index between the two reviewers (L.C. and A.S.) was 0.96 for data extraction, which indicated very good inter-observer agreement.

\section{Study characteristics}

Of the 90 studies reporting alcohol relapse, 86 were cohort studies (72 retrospective and 14 prospective cohorts), 2 were cross-sectional studies, and 2 were case-control studies (Table 3). Data for the 86 cohort studies were used for pooling in the incidence of alcohol relapse. Of these, 45 cohorts (40 retrospective and 5 prospective cohorts) were pooled for the proportion of heavy alcohol relapse; 37 studies (43.0\%) were from North America, 40 studies (46.5\%) were from Europe, 8 studies (9.3\%) were from Asia-Pacific, and 1 study $(0.1 \%)$ was from Brazil.

\section{The incidence of alcohol relapse}

The characteristics of the studies and the data on alcohol relapse rates are detailed in Table 3. A total of 86 cohort studies with 8061 patients reported incidences of alcohol relapse at any time after LT. The mean age of patients ranged from 35 to 60 years, and the mean follow-up time was 10 to 112 months. The alcohol relapse rate varied across studies with a range of 4 to $95 \%$ with an $\mathrm{I}^{2}$ of $90.7 \%$. A random effect model was applied and yielded the pooled alcohol relapse rate of $22 \%$ (95\% CI: 19-25\%) during the mean follow-up time of $48.4 \pm 24.7$ months. The rate of heavy alcohol relapse varied markedly across studies with an $\mathrm{I}^{2}$ of $85 \%$ and pooled rate of 14\% (95\%CI: $12-16 \%)$.

\section{Pooled risk factors of alcohol relapse}

The effects of all of the risk factors on alcohol relapse after LT that were classified by demographic, risk behavior, social, and comorbidity factors; these were pooled in 37 cohort studies (Table 4). The results of pooling these effects are summarized in Table 5 . The results showed that psychiatric comorbidities, pre-transplant abstinence less than 6 months, being unmarried, and smoking were significantly associated with alcohol relapse after LT with corresponding pooled ORs of 3.46 (95\% CI: 1.876.39), 2.76 (95\%CI: 2.10-3.61), 1.84 (95\%CI: 1.39-2.43), and 1.72 (95\%CI: $1.21-2.46)$, respectively. In addition, the $\mathrm{I}^{2}$ ranged from 0 to $40.6 \%$, with the highest $\mathrm{I}^{2}$ in psychiatric comorbidities.

\section{Subgroup analysis}

Subgroup analysis by age ( $\leq 50$ years or $>50$ years), regions of studies (Europe, North America, Asia Pacific and Brazil), definition of alcohol relapse (only report or report combining with biochemical testing), and followup time ( $\leq 4$ years or $>4$ years) was performed to explore the potential cause of heterogeneity of pooled rates of alcohol relapse and heavy alcohol relapse. Likewise, the subgroup analysis was performed with psychiatric comorbidities to identify the factor associated with alcohol relapse with the highest risk and heterogeneity. Subgroup analyses showed no significant difference in all analyses of alcohol relapse and heavy alcohol relapse rates except for one analysis of psychiatric comorbidities. 
Table 2 Newcastle-Ottawa quality assessment scale of each included studies

\begin{tabular}{|c|c|c|c|c|c|c|c|c|c|}
\hline Author & Year & S1 & S2 & S3 & S4 & C & O1 & $\mathrm{O} 2$ & $\mathrm{O} 3$ \\
\hline Starzl TE [30] & 1988 & * & * & & * & * & & & * \\
\hline Bird GLA [12] & 1990 & * & * & * & * & * & * & & * \\
\hline Kumar S [31] & 1990 & * & * & * & * & ** & * & & * \\
\hline Doffoel M[32] & 1992 & * & * & & * & * & & & * \\
\hline Knechtle SJ [33] & 1992 & * & * & * & * & * & * & * & \\
\hline Lucey MR [34] & 1992 & * & * & * & * & ** & * & * & * \\
\hline Berlakovich GA [35] & 1994 & * & * & * & * & * & * & & * \\
\hline Howard L [36] & 1994 & * & * & * & * & * & * & & * \\
\hline Osorio RW [37] & 1994 & * & * & * & * & ** & * & & * \\
\hline Gerhardt TC [38] & 1996 & * & * & * & * & ** & * & & * \\
\hline Tringali RA [39] & 1996 & * & * & * & * & ** & * & & * \\
\hline Tripp LE [40] & 1996 & * & * & & * & * & & & * \\
\hline Zibari GB [41] & 1996 & * & * & * & * & * & * & & * \\
\hline Anand AC [42] & 1997 & * & * & * & * & * & * & & $*$ \\
\hline Coffman KL [43] & 1997 & * & * & & * & * & & & * \\
\hline Everson G [44] & 1997 & * & * & * & * & * & * & & \\
\hline Foster PF [24] & 1997 & * & * & * & * & * & * & * & * \\
\hline Lucey MR [45] & 1997 & * & * & * & * & * & * & * & * \\
\hline Reeck UH [46] & 1997 & * & * & & * & * & & & \\
\hline Shakil AO [47] & 1997 & * & * & & * & * & & & * \\
\hline Stefanini GF [48] & 1997 & * & * & & * & * & & & * \\
\hline DiMartini A [49] & 1998 & * & * & & * & * & & & \\
\hline Fabrega E [50] & 1998 & * & * & * & * & * & * & & * \\
\hline Heinemann A [51] & 1998 & * & * & * & * & * & * & & * \\
\hline Tang H [52] & 1998 & * & * & & * & ** & & & * \\
\hline Conjeevaram HS [53] & 1999 & * & * & * & * & ** & * & & * \\
\hline Gledhill J [54] & 1999 & * & * & * & * & * & * & & * \\
\hline Newton SE [55] & 1999 & * & * & & * & * & & & \\
\hline Pageaux GP [18] & 1999 & * & * & * & * & ** & * & & * \\
\hline Romano DR [56] & 1999 & * & * & * & * & * & * & * & \\
\hline Abosh D [57] & 2000 & * & * & & * & * & & & \\
\hline Berlakovich GA [58] & 2000 & * & * & * & * & * & * & * & * \\
\hline Burra P [59] & 2000 & * & * & * & * & ** & * & & * \\
\hline DiMartini A [60] & 2000 & * & * & * & * & * & * & & * \\
\hline Jain A [61] & 2000 & * & * & * & * & ** & * & * & $\pi$ \\
\hline Pereira SP [62] & 2000 & * & * & * & * & * & * & & * \\
\hline Platz KP [21] & 2000 & * & * & * & * & * & * & & * \\
\hline Bellamy CO [63] & 2001 & * & * & * & * & * & * & & * \\
\hline DiMartini A [64] & 2001 & * & * & * & * & * & * & & * \\
\hline Gish RG [65] & 2001 & * & * & * & * & ** & * & * & * \\
\hline Karman JF [66] & 2001 & * & * & & * & * & & & * \\
\hline Mackie J [15] & 2001 & * & * & * & * & ** & * & & $\pi$ \\
\hline Tome S [67] & 2002 & * & * & & * & * & & & \\
\hline
\end{tabular}

Table 2 Newcastle-Ottawa quality assessment scale of each included studies (Continued)

\begin{tabular}{|c|c|c|c|c|c|c|c|c|c|}
\hline Author & Year & S1 & S2 & S3 & S4 & C & 01 & $\mathrm{O} 2$ & $\mathrm{O} 3$ \\
\hline Berlakovich GA [68] & 2004 & * & * & * & * & * & * & & * \\
\hline Jauhar S [14] & 2004 & * & * & & * & ** & & & * \\
\hline Miguet M [69] & 2004 & * & * & * & * & * & * & & * \\
\hline Björnsson E [20] & 2005 & * & * & * & * & *** & * & & * \\
\hline Cuadrado A [10] & 2005 & * & * & * & * & ** & * & * & * \\
\hline DiMartini A [70] & 2006 & * & * & * & * & * & * & & * \\
\hline Hwang S [71] & 2006 & * & * & * & * & * & * & & * \\
\hline Kelly M [72] & 2006 & * & * & * & * & *** & * & * & * \\
\hline De Gottardi A [29] & 2007 & * & * & * & * & *** & * & * & * \\
\hline Dumortier J [73] & 2007 & * & * & * & * & * & * & * & * \\
\hline Newton SE [74] & 2007 & * & * & & * & * & & * & \\
\hline Nickels M [75] & 2007 & * & * & & * & * & & & * \\
\hline Pfitzmann R [8] & 2007 & * & * & * & * & *** & * & * & * \\
\hline Vieira A [76] & 2007 & * & * & & * & * & & & * \\
\hline Wells JT [77] & 2007 & * & * & * & * & * & * & * & * \\
\hline Gedaly R [23] & 2008 & * & * & & * & * & & & * \\
\hline Immordino G [17] & 2009 & * & * & & * & * & & & * \\
\hline Tandon P [26] & 2009 & * & * & * & * & * & * & * & * \\
\hline Biselli M [78] & 2010 & * & * & * & * & * & * & * & * \\
\hline Chen GH [79] & 2010 & * & * & * & * & * & * & & * \\
\hline DiMartini A [80] & 2010 & * & * & * & * & * & * & & * \\
\hline Karim Z [81] & 2010 & * & * & * & * & *** & * & & * \\
\hline Hartl J [82] & 2011 & * & * & * & * & *** & * & & * \\
\hline Mathurin P [7] & 2011 & * & * & * & * & * & * & & * \\
\hline Schmeding M [9] & 2011 & * & * & * & * & * & * & & * \\
\hline Staufer K [83] & 2011 & * & * & * & * & * & * & & * \\
\hline Faure S [84] & 2012 & * & * & * & * & * & * & * & * \\
\hline Addolorato G [85] & 2013 & * & * & * & * & *** & * & & * \\
\hline Deruytter E [86] & 2013 & * & * & * & * & *** & * & * & * \\
\hline Kawaguchi Y [87] & 2013 & * & * & * & * & *** & * & & * \\
\hline Park YH [19] & 2013 & * & * & & * & *** & & * & * \\
\hline Rice JP [88] & 2013 & * & * & & * & *** & & * & * \\
\hline Rodrigue JR [89] & 2013 & * & * & * & * & *** & * & * & * \\
\hline Egawa H [16] & 2014 & * & * & * & * & *** & * & & * \\
\hline Grąt M [90] & 2014 & * & * & & * & * & & * & * \\
\hline Piano S [91] & 2014 & * & * & * & * & * & * & & * \\
\hline Dumortier J [92] & 2015 & * & * & * & * & * & * & * & * \\
\hline Hasanin M [93] & 2015 & * & * & & * & * & & * & \\
\hline Satapathy SK [94] & 2015 & * & * & & * & * & & * & * \\
\hline Zhou M [28] & 2015 & * & * & * & * & *** & * & & * \\
\hline Askgaard G [95] & 2016 & * & * & * & * & *** & * & & * \\
\hline Hajifathalian K [96] & 2016 & * & * & & * & * & & & * \\
\hline Im GY [97] & 2016 & * & * & & * & ** & & & * \\
\hline
\end{tabular}


Table 2 Newcastle-Ottawa quality assessment scale of each included studies (Continued)

\begin{tabular}{|c|c|c|c|c|c|c|c|c|c|}
\hline Author & Year & S1 & S2 & S3 & S4 & C & 01 & $\mathrm{O} 2$ & $\mathrm{O3}$ \\
\hline Kollmann D [98] & 2016 & * & * & * & * & *⿻丷木 & * & * & * \\
\hline Lee BP [99] & 2017 & * & * & * & * & ** & * & & * \\
\hline Onishi Y [100] & 2017 & * & * & * & * & *** & * & * & * \\
\hline Wigg AJ [101] & 2017 & * & * & * & * & ** & * & * & * \\
\hline
\end{tabular}

Patients with psychiatric comorbidities who had longer follow-up time ( $>4$ years) had an increased risk of alcohol relapse versus those with a shorter follow-up time ( $\leq 4$ years) (Fig. 2$)$.

\section{Publication bias}

The Egger test showed no evidence of publication bias among the studies, and the shape of the funnel plots was symmetrical in all analyses except for psychiatric comorbidities (Fig. 3) and abstinence less than 6 months (Fig. 4). The studies that reported less than 6 months of abstinence were both non-significant and significant leading to a contour-enhanced funnel plot; thus, asymmetry may not be due to either publication bias or heterogeneity. The studies with negative effect of psychiatric co-morbidities and abstinence less than 6 months were not reported.

\section{Discussion}

Alcohol relapse after LT remains an ethical issue in LT for alcoholic liver disease due to its harmful and negative impacts on liver grafts. One meta-analysis and four systemic reviews of alcoholic liver disease in LT patient were published earlier [25, 103-106]. The well-designed meta-analysis of rate and risk factors of alcohol relapse by Dew et al. in 2008 included 54 studies published between 1983 and 2005 [25]. A systematic review by Rustard et al. in 2015 selected only articles of the risk factors of alcohol relapse [103]. A systematic review by McCallum et al. in 2006 included only studies that were associated with psychosocial criteria [106]. Bravata et al. performed a systematic review of alcohol relapse and evaluated only the association between employment aspect and alcohol relapse [105]. One systematic review focused on neither alcohol relapse rate nor risk factor [104]. Our study is a systematic review and meta-analysis of all published studies up to 2018 , which aimed to estimate post-LT alcohol relapse rate and its predictive factors. To date, the current study is the most extensive meta-analysis of alcohol relapse in LT patients.

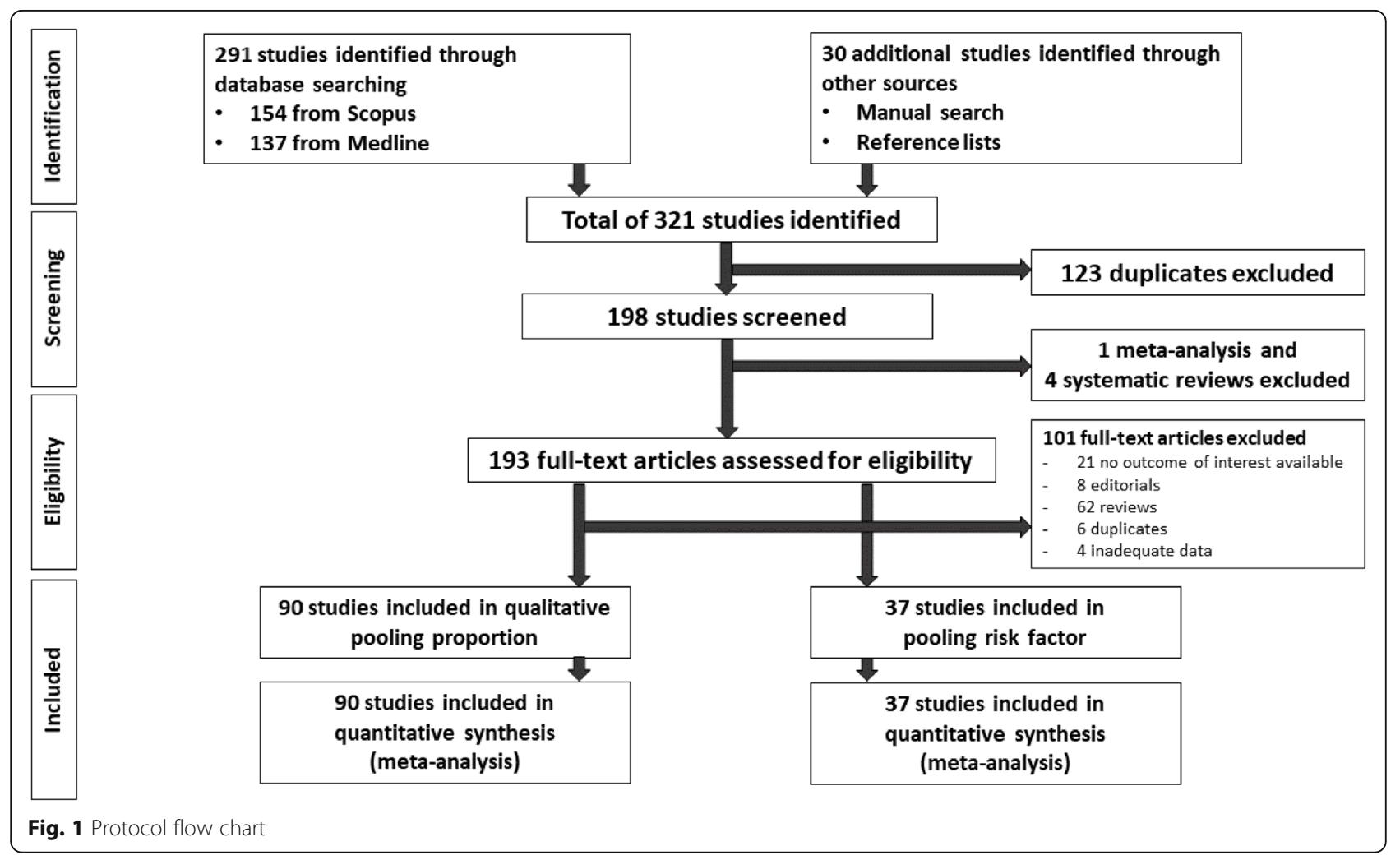


Table 3 Main characteristics of the studies included in the meta-analysis

\begin{tabular}{|c|c|c|c|c|c|c|c|c|}
\hline Author & Year & Type of study & $\begin{array}{l}\text { Region of } \\
\text { study }\end{array}$ & $\begin{array}{l}\text { Mean age } \\
\text { (years) }\end{array}$ & $\begin{array}{l}\text { Number of } \\
\text { population }\end{array}$ & $\begin{array}{l}\text { Number of any } \\
\text { relapse }\end{array}$ & $\begin{array}{l}\text { Number of heavy } \\
\text { relapse }\end{array}$ & $\begin{array}{l}\text { Follow-up time } \\
\text { (months) }\end{array}$ \\
\hline Starzl TE [30] & 1988 & $\begin{array}{l}\text { Retrospective } \\
\text { Cohort }\end{array}$ & US-Canada & - & 35 & 2 & - & 24 \\
\hline Bird GL [12] & 1990 & $\begin{array}{l}\text { Retrospective } \\
\text { Cohort }\end{array}$ & Europe & - & 18 & 4 & - & - \\
\hline Kumar S [31] & 1990 & $\begin{array}{l}\text { Retrospective } \\
\text { Cohort }\end{array}$ & US-Canada & 48.2 & 52 & 6 & 2 & 25 \\
\hline Doffoel M [32] & 1992 & $\begin{array}{l}\text { Retrospective } \\
\text { Cohort }\end{array}$ & Europe & - & 57 & 19 & - & - \\
\hline Knechtle SJ [33] & 1992 & Case-control & US-Canada & 51 & 32 & 4 & - & - \\
\hline Lucey MR [34] & 1992 & $\begin{array}{l}\text { Retrospective } \\
\text { Cohort }\end{array}$ & US-Canada & 44 & 45 & 5 & 2 & 15 \\
\hline $\begin{array}{l}\text { Berlakovich } \\
\text { GA[35] }\end{array}$ & 1994 & $\begin{array}{l}\text { Retrospective } \\
\text { Cohort }\end{array}$ & Europe & 47.6 & 44 & 14 & 7 & 33 \\
\hline Howard L [36] & 1994 & $\begin{array}{l}\text { Retrospective } \\
\text { Cohort }\end{array}$ & Europe & 50 & 20 & 19 & 16 & 34 \\
\hline Osorio RW [37] & 1994 & $\begin{array}{l}\text { Prospective } \\
\text { Cohort }\end{array}$ & US-Canada & 47 & 37 & 7 & 3 & 21 \\
\hline $\begin{array}{l}\text { Gerhardt TC } \\
\text { [38] }\end{array}$ & 1996 & $\begin{array}{l}\text { Retrospective } \\
\text { Cohort }\end{array}$ & US-Canada & 49 & 41 & 20 & 2 & 47 \\
\hline Tringali RA [39] & 1996 & $\begin{array}{l}\text { Retrospective } \\
\text { Cohort }\end{array}$ & US-Canada & 47.4 & 58 & 12 & 10 & 28 \\
\hline Tripp LE [40] & 1996 & $\begin{array}{l}\text { Retrospective } \\
\text { Cohort }\end{array}$ & US-Canada & 49 & 68 & 9 & 5 & - \\
\hline Zibari GB [41] & 1996 & $\begin{array}{l}\text { Retrospective } \\
\text { Cohort }\end{array}$ & US-Canada & 47 & 29 & 2 & - & - \\
\hline Anand AC [42] & 1997 & $\begin{array}{l}\text { Retrospective } \\
\text { Cohort }\end{array}$ & Europe & 47.5 & 39 & 5 & - & 25 \\
\hline Coffman KL [43] & 1997 & $\begin{array}{l}\text { Prospective } \\
\text { Cohort }\end{array}$ & US-Canada & - & 91 & 18 & - & - \\
\hline Everson G [44] & 1997 & $\begin{array}{l}\text { Retrospective } \\
\text { Cohort }\end{array}$ & US-Canada & 53 & 62 & 11 & 6 & 12 \\
\hline Foster PF [24] & 1997 & $\begin{array}{l}\text { Retrospective } \\
\text { Cohort }\end{array}$ & US-Canada & 48.6 & 63 & 13 & - & 49.3 \\
\hline Lucey MR [45] & 1997 & $\begin{array}{l}\text { Retrospective } \\
\text { Cohort }\end{array}$ & US-Canada & 46 & 50 & 17 & - & 63 \\
\hline Reeck UH [46] & 1997 & $\begin{array}{l}\text { Retrospective } \\
\text { Cohort }\end{array}$ & Europe & - & 52 & 6 & - & - \\
\hline Shakil AO [47] & 1997 & $\begin{array}{l}\text { Retrospective } \\
\text { Cohort }\end{array}$ & US-Canada & 41 & 8 & 3 & - & - \\
\hline $\begin{array}{l}\text { Stefanini GF } \\
{[48]}\end{array}$ & 1997 & $\begin{array}{l}\text { Retrospective } \\
\text { Cohort }\end{array}$ & Europe & 47 & 11 & 3 & 1 & 48 \\
\hline DiMartini A [49] & 1998 & $\begin{array}{l}\text { Retrospective } \\
\text { Cohort }\end{array}$ & US-Canada & 49.5 & 63 & 15 & - & - \\
\hline Fabrega E [50] & 1998 & $\begin{array}{l}\text { Retrospective } \\
\text { Cohort }\end{array}$ & Europe & 49 & 44 & 8 & - & 37.8 \\
\hline $\begin{array}{l}\text { Heinemann A } \\
\text { [51] }\end{array}$ & 1998 & $\begin{array}{l}\text { Retrospective } \\
\text { Cohort }\end{array}$ & Europe & 45.6 & 13 & 4 & - & - \\
\hline Tang H [52] & 1998 & $\begin{array}{l}\text { Retrospective } \\
\text { Cohort }\end{array}$ & Europe & 48 & 56 & 28 & 9 & 24 \\
\hline $\begin{array}{l}\text { Conjeevaram } \\
\text { HS [53] }\end{array}$ & 1999 & $\begin{array}{l}\text { Retrospective } \\
\text { Cohort }\end{array}$ & US-Canada & 47 & 68 & 8 & 8 & - \\
\hline Gledhill J [54] & 1999 & $\begin{array}{l}\text { Retrospective } \\
\text { Cohort }\end{array}$ & Europe & 48 & 31 & 7 & - & 13.5 \\
\hline
\end{tabular}


Table 3 Main characteristics of the studies included in the meta-analysis (Continued)

\begin{tabular}{|c|c|c|c|c|c|c|c|c|}
\hline Author & Year & Type of study & $\begin{array}{l}\text { Region of } \\
\text { study }\end{array}$ & $\begin{array}{l}\text { Mean age } \\
\text { (years) }\end{array}$ & $\begin{array}{l}\text { Number of } \\
\text { population }\end{array}$ & $\begin{array}{l}\text { Number of any } \\
\text { relapse }\end{array}$ & $\begin{array}{l}\text { Number of heavy } \\
\text { relapse }\end{array}$ & $\begin{array}{l}\text { Follow-up time } \\
\text { (months) }\end{array}$ \\
\hline Newton SE [55] & 1999 & $\begin{array}{l}\text { Retrospective } \\
\text { Cohort }\end{array}$ & US-Canada & 47 & 122 & 33 & - & 62 \\
\hline $\begin{array}{l}\text { Pageaux GP } \\
{[18]}\end{array}$ & 1999 & $\begin{array}{l}\text { Retrospective } \\
\text { Cohort }\end{array}$ & Europe & 48.8 & 47 & 15 & 5 & 42.1 \\
\hline $\begin{array}{l}\text { Romano DR } \\
\text { [56] }\end{array}$ & 1999 & Case-control & Europe & 47.6 & 152 & 7 & - & - \\
\hline Abosh D [57] & 2000 & $\begin{array}{l}\text { Retrospective } \\
\text { Cohort }\end{array}$ & US-Canada & 50 & 10 & 5 & 5 & 10 \\
\hline $\begin{array}{l}\text { Berlakovich GA } \\
\text { [58] }\end{array}$ & 2000 & $\begin{array}{l}\text { Retrospective } \\
\text { Cohort }\end{array}$ & Europe & - & 118 & 15 & - & 53.7 \\
\hline Burra P [59] & 2000 & $\begin{array}{l}\text { Prospective } \\
\text { Cohort }\end{array}$ & Asia Pacific & 48 & 34 & 11 & 4 & 40.1 \\
\hline DiMartini A [60] & 2000 & $\begin{array}{l}\text { Retrospective } \\
\text { Cohort }\end{array}$ & Europe & 50 & 72 & 4 & 1 & - \\
\hline Jain A [61] & 2000 & $\begin{array}{l}\text { Retrospective } \\
\text { Cohort }\end{array}$ & US-Canada & 50.8 & 185 & 37 & 1 & 94 \\
\hline Pereira SP [62] & 2000 & $\begin{array}{l}\text { Retrospective } \\
\text { Cohort }\end{array}$ & Europe & 51 & 56 & 28 & 15 & 30 \\
\hline Platz KP [21] & 2000 & $\begin{array}{l}\text { Retrospective } \\
\text { Cohort }\end{array}$ & Europe & - & 117 & 30 & - & - \\
\hline Bellamy CO [63] & 2001 & $\begin{array}{l}\text { Retrospective } \\
\text { Cohort }\end{array}$ & US-Canada & 53 & 123 & 13 & - & 84 \\
\hline DiMartini A [64] & 2001 & $\begin{array}{l}\text { Prospective } \\
\text { Cohort }\end{array}$ & US-Canada & - & 36 & 8 & - & - \\
\hline Gish RG [65] & 2001 & $\begin{array}{l}\text { Prospective } \\
\text { Cohort }\end{array}$ & US-Canada & 47 & 61 & 12 & - & 82.8 \\
\hline Karman JF [66] & 2001 & $\begin{array}{l}\text { Retrospective } \\
\text { Cohort }\end{array}$ & US-Canada & 49 & 19 & 4 & - & 36 \\
\hline Mackie J [15] & 2001 & $\begin{array}{l}\text { Retrospective } \\
\text { Cohort }\end{array}$ & Europe & 51 & 46 & 21 & 3 & 25 \\
\hline Tome S [67] & 2002 & $\begin{array}{l}\text { Prospective } \\
\text { Cohort }\end{array}$ & Europe & 51 & 68 & 7 & 2 & 38 \\
\hline $\begin{array}{l}\text { Berlakovich GA } \\
\text { [68] }\end{array}$ & 2004 & Case-control & Europe & 51.5 & 44 & 3 & - & 43.5 \\
\hline Jauhar S [14] & 2004 & $\begin{array}{l}\text { Retrospective } \\
\text { Cohort }\end{array}$ & US-Canada & 51 & 11 & 17 & 15 & 44.1 \\
\hline Miguet M [69] & 2004 & $\begin{array}{l}\text { Prospective } \\
\text { Cohort }\end{array}$ & Europe & 48.7 & 51 & 13 & 9 & 35.7 \\
\hline Björnsson E [20] & 2005 & $\begin{array}{l}\text { Retrospective } \\
\text { Cohort }\end{array}$ & Europe & 53 & 93 & 32 & 7 & 31 \\
\hline $\begin{array}{l}\text { Cuadrado A } \\
\text { [10] }\end{array}$ & 2005 & $\begin{array}{l}\text { Retrospective } \\
\text { Cohort }\end{array}$ & Europe & 48.9 & 54 & 14 & 14 & 99.2 \\
\hline DiMartini A [70] & 2006 & $\begin{array}{l}\text { Prospective } \\
\text { Cohort }\end{array}$ & US-Canada & 49.7 & 167 & 70 & 43 & - \\
\hline Hwang S [71] & 2006 & $\begin{array}{l}\text { Retrospective } \\
\text { Cohort }\end{array}$ & US-Canada & 50 & 15 & 3 & - & 41 \\
\hline Kelly M [72] & 2006 & $\begin{array}{l}\text { Retrospective } \\
\text { Cohort }\end{array}$ & Asia Pacific & 50.1 & 90 & 28 & 18 & 67 \\
\hline $\begin{array}{l}\text { De Gottardi A } \\
\text { [29] }\end{array}$ & 2007 & $\begin{array}{l}\text { Retrospective } \\
\text { Cohort }\end{array}$ & Europe & 51.3 & 387 & 46 & 46 & 61.2 \\
\hline $\begin{array}{l}\text { Dumortier J } \\
\text { [73] }\end{array}$ & 2007 & $\begin{array}{l}\text { Retrospective } \\
\text { Cohort }\end{array}$ & Europe & 50 & 305 & 37 & 37 & 63 \\
\hline Newton SE [74] & 2007 & Cross sectional & US-Canada & - & 18 & 4 & - & - \\
\hline
\end{tabular}


Table 3 Main characteristics of the studies included in the meta-analysis (Continued)

\begin{tabular}{|c|c|c|c|c|c|c|c|c|}
\hline Author & Year & Type of study & $\begin{array}{l}\text { Region of } \\
\text { study }\end{array}$ & $\begin{array}{l}\text { Mean age } \\
\text { (years) }\end{array}$ & $\begin{array}{l}\text { Number of } \\
\text { population }\end{array}$ & $\begin{array}{l}\text { Number of any } \\
\text { relapse }\end{array}$ & $\begin{array}{l}\text { Number of heavy } \\
\text { relapse }\end{array}$ & $\begin{array}{l}\text { Follow-up time } \\
\text { (months) }\end{array}$ \\
\hline Nickels M [75] & 2007 & $\begin{array}{l}\text { Retrospective } \\
\text { Cohort }\end{array}$ & US-Canada & 48.8 & 27 & 8 & - & 23.4 \\
\hline Pfitzmann R [8] & 2007 & $\begin{array}{l}\text { Retrospective } \\
\text { Cohort }\end{array}$ & Europe & 51.2 & 290 & 56 & 23 & 89 \\
\hline Vieira A [76] & 2007 & $\begin{array}{l}\text { Retrospective } \\
\text { Cohort }\end{array}$ & Other(Brazil) & 47 & 17 & 2 & - & 29.6 \\
\hline Wells JT [77] & 2007 & $\begin{array}{l}\text { Retrospective } \\
\text { Cohort }\end{array}$ & Europe & 50.3 & 148 & 24 & 20 & 90.5 \\
\hline Gedaly R [23] & 2008 & $\begin{array}{l}\text { Retrospective } \\
\text { Cohort }\end{array}$ & US-Canada & 52 & 142 & 27 & - & 41.2 \\
\hline $\begin{array}{l}\text { Immordino G } \\
{[17]}\end{array}$ & 2009 & $\begin{array}{l}\text { Retrospective } \\
\text { Cohort }\end{array}$ & US-Canada & 53.2 & 110 & 13 & - & - \\
\hline Tandon P [26] & 2009 & $\begin{array}{l}\text { Retrospective } \\
\text { Cohort }\end{array}$ & US-Canada & 52 & 171 & 41 & 22 & 64.8 \\
\hline Biselli M [78] & 2010 & $\begin{array}{l}\text { Retrospective } \\
\text { Cohort }\end{array}$ & Europe & 48 & 49 & 13 & 3 & 58 \\
\hline Chen GH [79] & 2010 & $\begin{array}{l}\text { Retrospective } \\
\text { Cohort }\end{array}$ & Asia Pacific & 52.8 & 16 & 1 & - & 32.4 \\
\hline DiMartini A [80] & 2010 & $\begin{array}{l}\text { Prospective } \\
\text { Cohort }\end{array}$ & US-Canada & 52 & 208 & 95 & - & - \\
\hline Karim Z [81] & 2010 & $\begin{array}{l}\text { Retrospective } \\
\text { Cohort }\end{array}$ & US-Canada & 50.5 & 80 & 8 & 8 & - \\
\hline Hartl J [82] & 2011 & $\begin{array}{l}\text { Retrospective } \\
\text { Cohort }\end{array}$ & Europe & 52.5 & 109 & 17 & - & 31 \\
\hline Mathurin P [7] & 2011 & $\begin{array}{l}\text { Prospective } \\
\text { Cohort }\end{array}$ & Europe & 47.4 & 26 & 3 & 2 & 20 \\
\hline $\begin{array}{l}\text { Schmeding M } \\
\text { [9] }\end{array}$ & 2011 & $\begin{array}{l}\text { Retrospective } \\
\text { Cohort }\end{array}$ & Europe & 48.9 & 271 & 73 & 73 & - \\
\hline Staufer K [83] & 2011 & $\begin{array}{l}\text { Prospective } \\
\text { Cohort }\end{array}$ & Europe & 53.5 & 141 & 28 & - & - \\
\hline Faure S [84] & 2012 & $\begin{array}{l}\text { Retrospective } \\
\text { Cohort }\end{array}$ & Europe & 51 & 206 & 90 & 50 & 81.7 \\
\hline $\begin{array}{l}\text { Addolorato G } \\
\text { [85] }\end{array}$ & 2013 & $\begin{array}{l}\text { Retrospective } \\
\text { Cohort }\end{array}$ & Europe & 49.4 & 92 & 22 & - & - \\
\hline Deruytter E [86] & 2013 & $\begin{array}{l}\text { Retrospective } \\
\text { Cohort }\end{array}$ & Europe & 56 & 108 & 31 & 17 & 55 \\
\hline $\begin{array}{l}\text { Kawaguchi Y } \\
\text { [87] }\end{array}$ & 2013 & $\begin{array}{l}\text { Retrospective } \\
\text { Cohort }\end{array}$ & Asia Pacific & 52 & 13 & 1 & - & 38 \\
\hline Park YH [19] & 2013 & $\begin{array}{l}\text { Retrospective } \\
\text { Cohort }\end{array}$ & Asia Pacific & 52 & 18 & 3 & 2 & 57 \\
\hline Rice JP [88] & 2013 & $\begin{array}{l}\text { Retrospective } \\
\text { Cohort }\end{array}$ & US-Canada & 49.3 & 300 & 48 & 16 & 82 \\
\hline $\begin{array}{l}\text { Rodrigue JR } \\
\text { [107] }\end{array}$ & 2013 & $\begin{array}{l}\text { Retrospective } \\
\text { Cohort }\end{array}$ & US-Canada & 55 & 118 & 40 & 12 & 55 \\
\hline Egawa H [16] & 2014 & $\begin{array}{l}\text { Retrospective } \\
\text { Cohort }\end{array}$ & Asia Pacific & 35 & 140 & 32 & 21 & 44 \\
\hline Grąt M [90] & 2014 & $\begin{array}{l}\text { Retrospective } \\
\text { Cohort }\end{array}$ & Europe & 46 & 66 & 22 & - & 88.8 \\
\hline Piano S [91] & 2014 & $\begin{array}{l}\text { Prospective } \\
\text { Cohort }\end{array}$ & Europe & 60 & 23 & 5 & - & - \\
\hline $\begin{array}{l}\text { Dumortier J } \\
\text { [92] }\end{array}$ & 2015 & $\begin{array}{l}\text { Retrospective } \\
\text { Cohort }\end{array}$ & Europe & 47.2 & 712 & 128 & 128 & 63 \\
\hline Hasanin M [93] & 2015 & Cross-sectional & US-Canada & - & 45 & 8 & - & - \\
\hline
\end{tabular}


Table 3 Main characteristics of the studies included in the meta-analysis (Continued)

\begin{tabular}{|c|c|c|c|c|c|c|c|c|}
\hline Author & Year & Type of study & $\begin{array}{l}\text { Region of } \\
\text { study }\end{array}$ & $\begin{array}{l}\text { Mean age } \\
\text { (years) }\end{array}$ & $\begin{array}{l}\text { Number of } \\
\text { population }\end{array}$ & $\begin{array}{l}\text { Number of any } \\
\text { relapse }\end{array}$ & $\begin{array}{l}\text { Number of heavy } \\
\text { relapse }\end{array}$ & $\begin{array}{l}\text { Follow-up time } \\
\text { (months) }\end{array}$ \\
\hline $\begin{array}{l}\text { Satapathy SK } \\
\text { [94] }\end{array}$ & 2015 & $\begin{array}{l}\text { Retrospective } \\
\text { Cohort }\end{array}$ & US-Canada & 54 & 148 & 16 & 16 & 112.8 \\
\hline Zhou M [28] & 2015 & $\begin{array}{l}\text { Retrospective } \\
\text { Cohort }\end{array}$ & US-Canada & 54.2 & 35 & 6 & - & - \\
\hline Askgaard G [95] & 2016 & $\begin{array}{l}\text { Retrospective } \\
\text { Cohort }\end{array}$ & Europe & 54 & 156 & 35 & 35 & - \\
\hline $\begin{array}{l}\text { Hajifathalian K } \\
\text { [96] }\end{array}$ & 2016 & $\begin{array}{l}\text { Prospective } \\
\text { Cohort }\end{array}$ & Europe & 56 & 19 & 4 & - & 40.8 \\
\hline Im GY [97] & 2016 & $\begin{array}{l}\text { Retrospective } \\
\text { Cohort }\end{array}$ & US-Canada & 41 & 9 & 2 & 1 & 24.5 \\
\hline Kollmann D [98] & 2016 & $\begin{array}{l}\text { Retrospective } \\
\text { Cohort }\end{array}$ & Europe & - & 382 & 16 & - & 73 \\
\hline Lee BP [99] & 2017 & $\begin{array}{l}\text { Retrospective } \\
\text { Cohort }\end{array}$ & US-Canada & 51.4 & 31 & 11 & 7 & 19.2 \\
\hline Onishi Y [100] & 2017 & $\begin{array}{l}\text { Retrospective } \\
\text { Cohort }\end{array}$ & Asia Pacific & 46 & 7 & 1 & - & 60 \\
\hline Wigg AJ [101] & 2017 & $\begin{array}{l}\text { Retrospective } \\
\text { Cohort }\end{array}$ & Asia Pacific & 50 & 87 & 18 & 14 & 52 \\
\hline
\end{tabular}

Our study demonstrated that any alcohol relapse and heavy alcohol relapse rates were as high as 22 and $14 \%$ during the mean follow-up time of $48.4 \pm$ 24.7 months, respectively. The literature on alcohol relapse post-transplantation has reported a wide range of alcohol relapse rates, which might be due to different definitions of alcohol relapse. Dew et al. reported that the average rate for alcohol relapse after LT was 5.6 cases per 100 patients per year for any alcohol relapse and 2.5 cases per 100 patients per year for heavy alcohol relapse [25]. The authors suggested that a significant proportion of patients who returned to any alcohol drinking then became heavy drinkers, which led to a significant harm to LT recipients [25].

In our study, the most significant risk factors of relapse were psychiatric comorbidities followed by pre-transplant alcohol abstinence less than 6 months, being unmarried, and smoking. Four of the nine studies reported that psychiatric conditions had a link with alcohol relapse $[16,29,72,81]$. The finding was consistent with the previous meta-analysis [25]. The study identified 3 of the 12 psychosocial variables associated with any alcohol relapse: $<6$ months abstinence prior to transplant, poor social support, and a family history of alcohol abuse or dependence [25]. We found that 9 of the 20 studies revealed that alcohol abstinence less than 6 months was associated with alcohol relapse $[8,18,29,37$, $81,82,95,107,108]$. Our study confirms the validity of using the 6-month rule of alcohol abstinence as a criterion for pre-transplant selection in patients with ALD; this is consistent with the previous metaanalysis study [25].

A systematic review of large prospective studies focusing on risk factors for alcohol relapse following LT has also suggested that a shorter length of pre-transplant sobriety was a significant predictor of alcohol relapse [103]. However, the 6-month rule cannot be applied in LT for patients with severe acute alcoholic hepatitis whose condition is not allowed to wait until 6 months. LT in this group of patients remains a controversial issue in many transplant centers. The current data do not suggest that LT in patients with severe alcoholic hepatitis leads to more alcohol relapse [109]. Therefore, 6 months of alcohol abstinence may not reliably predict post-LT alcohol relapse. Other risk factors were psychiatric comorbidities, a high score on the HRAR scale, and a diagnosis of alcohol dependence [103]. Scoring systems to predict alcohol relapse after LT such as HRAR and the ARRA were proposed for use, but they have never been validated by welldesigned studies.

In this study, psychiatric co-morbidities and pretransplant abstinence less than 6 months were strong predictive factors of alcohol relapse with some publication bias against negative studies. Psychiatric comorbidities were the strongest risk factor in this study but with high heterogeneity. Interestingly, subsequent subgroup analysis showed that longer follow-up times led to an increased impact 
Table 4 Summary of the included studies reported risk factors in the meta-analysis

\begin{tabular}{|c|c|c|c|c|c|}
\hline Author & Year & Demographic factors & Risk behavior factors & Social factors & Comorbidity \\
\hline Kumar S [31] & 1990 & - & - & Abstinence $<6$ months & - \\
\hline Osorio RW [37] & 1994 & $\begin{array}{l}\text { Male } \\
\text { Unmarried } \\
\text { Unemployed }\end{array}$ & Substance use & $\begin{array}{l}\text { Abstinence }<6 \text { months } \\
\text { Rehabilitation }\end{array}$ & Psychiatric disease \\
\hline Gerhardt TC [38] & 1996 & - & - & Abstinence $<6$ months & - \\
\hline Tringali RA [39] & 1996 & - & - & Abstinence $<6$ months & - \\
\hline Foster PF [24] & 1997 & Family history of alcohol use & Substance use & $\begin{array}{l}\text { Abstinence }<6 \text { months } \\
\text { Rehabilitation }\end{array}$ & - \\
\hline Lucey MR [45] & 1997 & Male & - & Abstinence $<6$ months & - \\
\hline Shakil AO [47] & 1997 & Male & - & - & - \\
\hline Tang H [52] & 1998 & Male & - & - & - \\
\hline Conjeevaram HS [53] & 1999 & Male & - & - & - \\
\hline Newton SE [55] & 1999 & - & Substance use & - & - \\
\hline Pageaux GP [18] & 1999 & $\begin{array}{l}\text { Male } \\
\text { Unmarried } \\
\text { Unemployed }\end{array}$ & - & Abstinence $<6$ months & - \\
\hline Burra P [59] & 2000 & $\begin{array}{l}\text { Unmarried } \\
\text { Family history of alcohol use }\end{array}$ & $\begin{array}{l}\text { Substance use } \\
\text { Alcohol dependence }\end{array}$ & Rehabilitation & - \\
\hline Jain A [61] & 2000 & - & - & $\begin{array}{l}\text { Abstinence }<6 \text { months } \\
\text { Rehabilitation }\end{array}$ & - \\
\hline Mackie J [15] & 2001 & $\begin{array}{l}\text { Male } \\
\text { Unmarried } \\
\text { Lack of social support } \\
\text { Low SES } \\
\text { Family history of alcohol use }\end{array}$ & Smoking & Abstinence $<6$ months & - \\
\hline Jauhar S [14] & 2004 & $\begin{array}{l}\text { Male } \\
\text { Unmarried } \\
\text { Unemployed }\end{array}$ & Substance use & $\begin{array}{l}\text { Abstinence }<6 \text { months } \\
\text { Rehabilitation }\end{array}$ & Psychiatric disease \\
\hline Björnsson E [20] & 2005 & - & - & Rehabilitation & - \\
\hline Cuadrado A [10] & 2005 & Male & - & & - \\
\hline Hwang S [71] & 2006 & - & - & Abstinence $<6$ months & - \\
\hline Kelly M [72] & 2006 & $\begin{array}{l}\text { Unmarried } \\
\text { Lack of social support } \\
\text { Unemployed }\end{array}$ & Substance use & Abstinence $<6$ months & $\begin{array}{l}\text { Psychiatric disease } \\
\text { Depression }\end{array}$ \\
\hline De Gottardi A [29] & 2007 & $\begin{array}{l}\text { Age }<50 \text { years } \\
\text { Male } \\
\text { Unmarried } \\
\text { Low SES } \\
\text { Unemployed }\end{array}$ & High HRAR & Abstinence $<6$ months & Psychiatric disease \\
\hline Nickels M [75] & 2007 & $\begin{array}{l}\text { Age }<50 \text { years } \\
\text { Male }\end{array}$ & Alcohol dependence & - & Depression \\
\hline Pfitzmann R [8] & 2007 & $\begin{array}{l}\text { Age }<50 \text { years } \\
\text { Male } \\
\text { Unmarried }\end{array}$ & - & Abstinence $<6$ months & - \\
\hline Karim Z [81] & 2010 & $\begin{array}{l}\text { Age }<50 \text { years } \\
\text { Male } \\
\text { Unmarried } \\
\text { Low SES } \\
\text { Unemployed }\end{array}$ & $\begin{array}{l}\text { Smoking } \\
\text { Substance use }\end{array}$ & $\begin{array}{l}\text { Abstinence }<6 \text { months } \\
\text { Rehabilitation }\end{array}$ & Psychiatric disease \\
\hline Hartl J [82] & 2011 & - & Smoking & $\begin{array}{l}\text { Abstinence }<6 \text { months } \\
\text { Rehabilitation }\end{array}$ & - \\
\hline Addolorato G [85] & 2013 & - & - & Rehabilitation & - \\
\hline Deruytter E [86] & 2013 & $\begin{array}{l}\text { Age }<50 \text { years } \\
\text { Male } \\
\text { Unmarried } \\
\text { Unemployed } \\
\text { Family history of alcohol use }\end{array}$ & $\begin{array}{l}\text { Smoking } \\
\text { Alcohol dependence }\end{array}$ & - & Psychiatric disease \\
\hline Kawaguchi Y [87] & 2013 & Male & High HRAR & - & - \\
\hline Park YH [19] & 2013 & Male & - & Abstinence $<6$ months & - \\
\hline Rice JP [88] & 2013 & Male & - & - & - \\
\hline
\end{tabular}


Table 4 Summary of the included studies reported risk factors in the meta-analysis (Continued)

\begin{tabular}{|c|c|c|c|c|c|}
\hline Author & Year & Demographic factors & Risk behavior factors & Social factors & Comorbidity \\
\hline $\begin{array}{l}\text { Rodrigue JR [89] } \\
\text { no }\end{array}$ & 2013 & Lack of social support & Smoking & $\begin{array}{l}\text { Abstinence < 6months } \\
\text { Rehabilitation }\end{array}$ & - \\
\hline Egawa H [16] & 2014 & $\begin{array}{l}\text { Male } \\
\text { Unmarried } \\
\text { Lack of social support } \\
\text { Unemployed }\end{array}$ & $\begin{array}{l}\text { Smoking } \\
\text { High HRAR }\end{array}$ & Abstinence $<6$ months & Psychiatric disease \\
\hline Zhou M [28] & 2015 & - & High HRAR & - & - \\
\hline Askgaard G [95] & 2016 & $\begin{array}{l}\text { Male } \\
\text { Unmarried } \\
\text { Unemployed } \\
\text { Family history of alcohol use }\end{array}$ & $\begin{array}{l}\text { Smoking } \\
\text { Alcohol dependence }\end{array}$ & Abstinence $<6$ months & Depression \\
\hline Im GY [97] & 2016 & $\begin{array}{l}\text { Male } \\
\text { Unmarried } \\
\text { Family history of alcohol use }\end{array}$ & Smoking & - & Psychiatric disease \\
\hline Lee BP [99] & 2017 & Male & - & - & - \\
\hline Onishi Y [100] & 2017 & $\begin{array}{l}\text { Age }<50 \text { years } \\
\text { Male }\end{array}$ & - & - & - \\
\hline Wigg AJ [101] & 2017 & $\begin{array}{l}\text { Male } \\
\text { Unmarried } \\
\text { Lack of social support } \\
\text { Unemployed } \\
\text { Family history of alcohol use }\end{array}$ & $\begin{array}{l}\text { Smoking } \\
\text { Substance use }\end{array}$ & Rehabilitation & Psychiatric disease \\
\hline
\end{tabular}

of psychiatric comorbidities on any alcohol relapse. The psychiatric comorbidities defined in enrolled studies included all psychiatric conditions that could cause impaired daily functioning, i.e. anxiety, schizophrenia, and personality disorders. In this study, we analyzed three cohort studies that reported depression separately because depression is a known risk factor associated with alcoholic drinking. We found that depression was not a significant factor in alcohol relapse $(\mathrm{OR}=3$; 95\%CI 0.49-9.25).

Table 5 Pooled risk factors of alcohol relapse

\begin{tabular}{|c|c|c|c|c|c|c|}
\hline Factors & $\mathrm{N}$ & OR & $95 \% \mathrm{Cl}$ & Pooling method & $1^{2}$ & Egger test (P-value) \\
\hline \multicolumn{7}{|l|}{ Demographic factors } \\
\hline Age $<50$ years & 6 & 1.16 & $0.43-3.15$ & Random effect & 75.2 & 0.55 \\
\hline Sex (male) & 23 & 0.89 & $0.69-1.11$ & Fixed effect & 21.7 & 0.43 \\
\hline Unmarried & 14 & 1.84 & $1.39-2.43$ & Fixed effect & 14.6 & 0.57 \\
\hline Lack of social support & 5 & 1.78 & $0.72-4.38$ & Random effect & 49.5 & 0.18 \\
\hline LOW SES & 3 & 0.99 & $0.15-6.50$ & Random effect & 86.3 & 0.28 \\
\hline Unemployed & 10 & 1.33 & $0.93-1.89$ & Fixed effect & 7.7 & 0.74 \\
\hline Family history of alcohol use & 7 & 1.49 & $0.94-2.36$ & Fixed effect & 23.0 & 0.50 \\
\hline \multicolumn{7}{|l|}{ Risk behavior factors } \\
\hline Smoking & 9 & 1.72 & $1.21-2.46$ & Fixed effect & 0 & 0.69 \\
\hline Substance use & 8 & 1.06 & $0.48-2.34$ & Random effect & 58.5 & 0.71 \\
\hline Alcohol dependence & 4 & 1.22 & $0.43-3.40$ & Random effect & 61.8 & 0.15 \\
\hline High HRAR & 4 & 2.93 & $0.30-28.64$ & Random effect & 79.6 & 0.18 \\
\hline \multicolumn{7}{|l|}{ Social factors } \\
\hline Abstinence $<6$ months & 20 & 2.76 & $2.10-3.61$ & Fixed effect & 18.1 & 0.02 \\
\hline Rehabilitation program & 11 & 1.10 & $0.59-2.04$ & Random effect & 67 & 0.71 \\
\hline \multicolumn{7}{|l|}{ Comorbidity } \\
\hline Psychiatric disease & 9 & 3.46 & $1.87-6.39$ & Random effect & 40.6 & 0.02 \\
\hline Depression & 3 & 2.13 & $0.49-9.25$ & Random effect & 54.4 & 0.60 \\
\hline
\end{tabular}

$N$ Number, OR Odds ratio, CI Confidence interval, $I^{2} I^{2}$ statistics, SES Socioeconomic status, HRAR High-risk alcohol relapse scale 


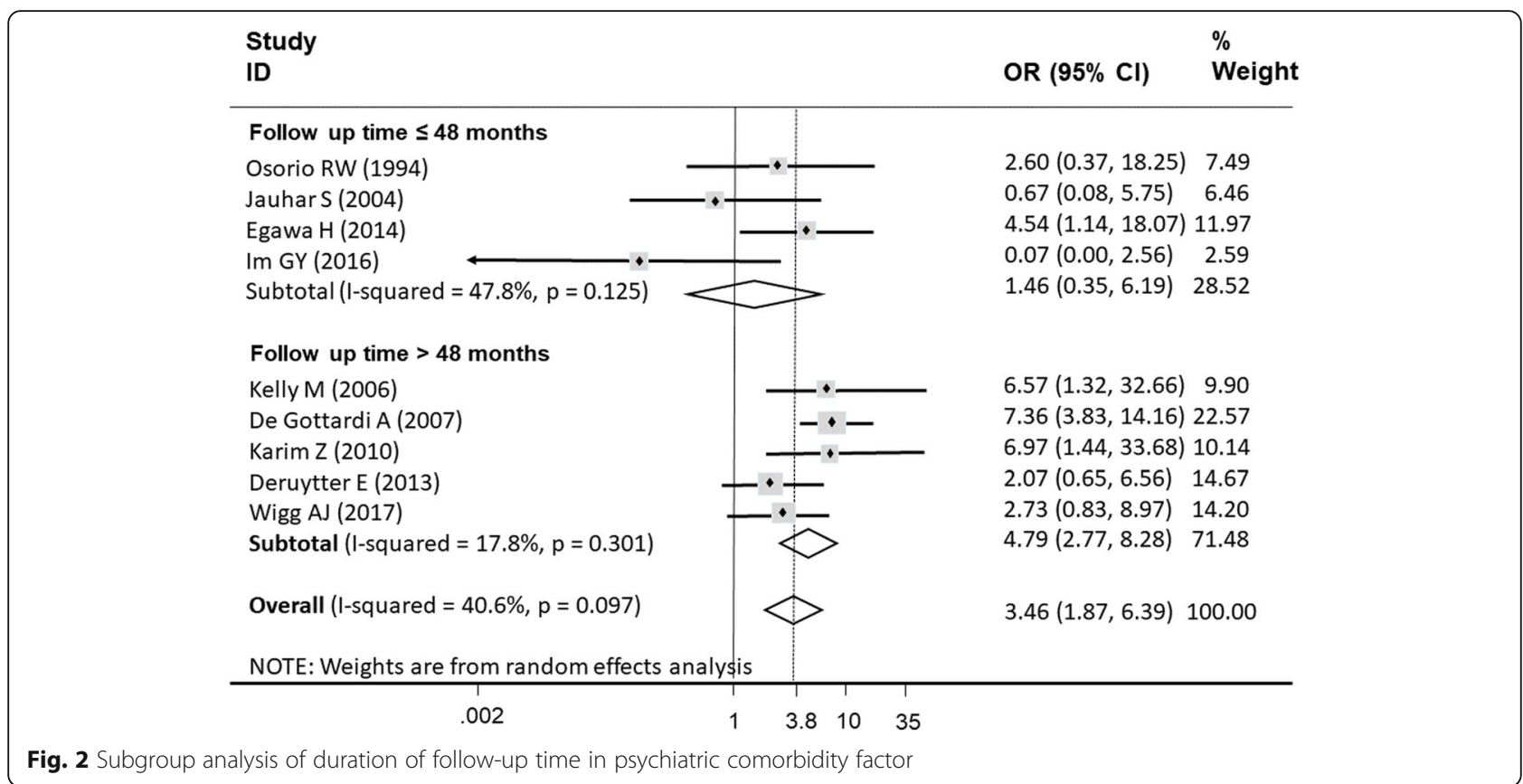

Clinical practice has changed considerably since the first studies that recruited in 1988. The differences in the definition of alcohol relapse and heavy relapse as well as a lack of objective means of documenting alcohol use in these studies are limitations. Furthermore, heavy alcohol relapse was defined only in some studies (Table 2). Including unpublished studies may solve this problem. The absence of negative studies of psychiatric co-morbidities and abstinence less than 6 months likely caused publication bias. However, this attempt cannot guarantee a reasonably low heterogeneity after including unpublished studies.

\section{Conclusions}

We demonstrated the pooled rates of any alcohol relapse and heavy alcohol relapse post-LT. Furthermore, we identified predictive factors of alcohol relapse after LT to be used during the selection process of $\mathrm{LT}$ candidates. With respect to the prevention of alcohol relapse post-LT, alcohol abstinence of at least 6 months, appropriate screening and

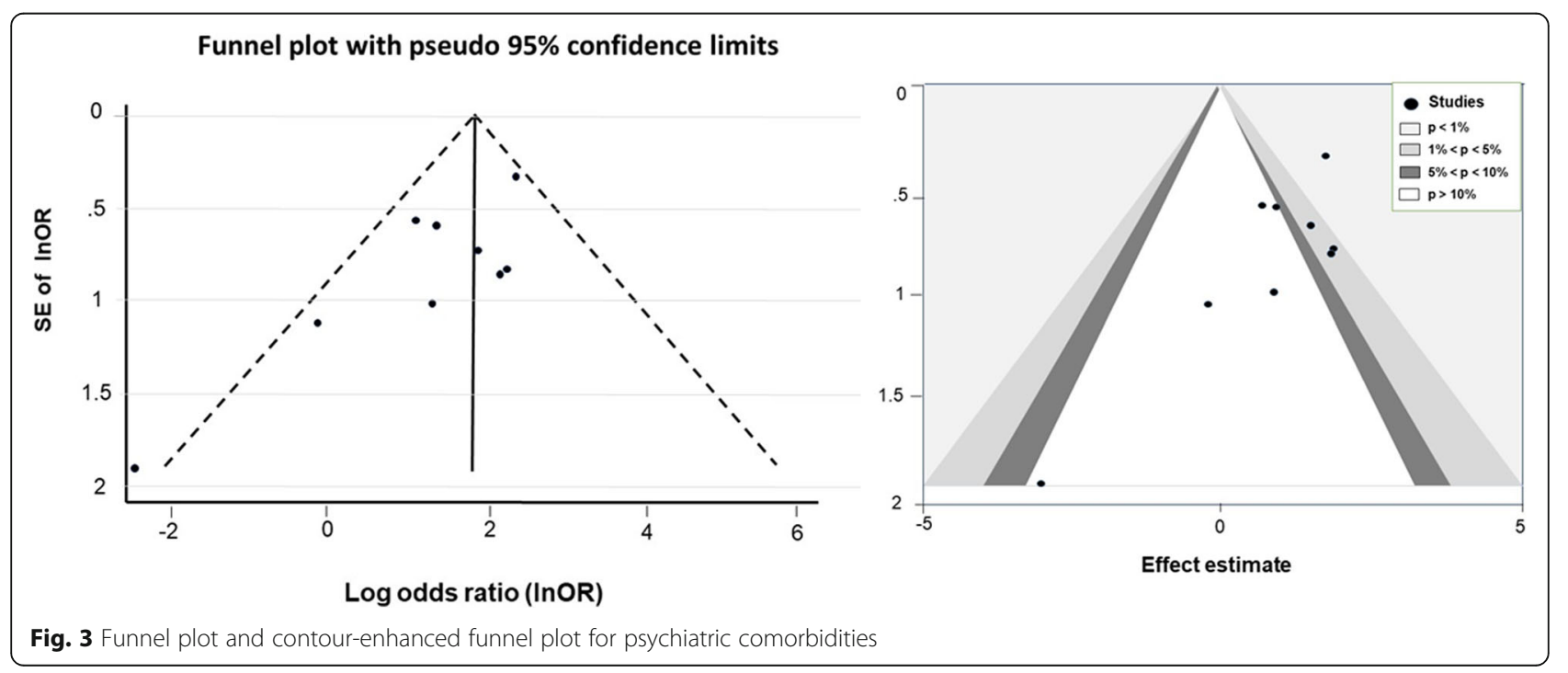



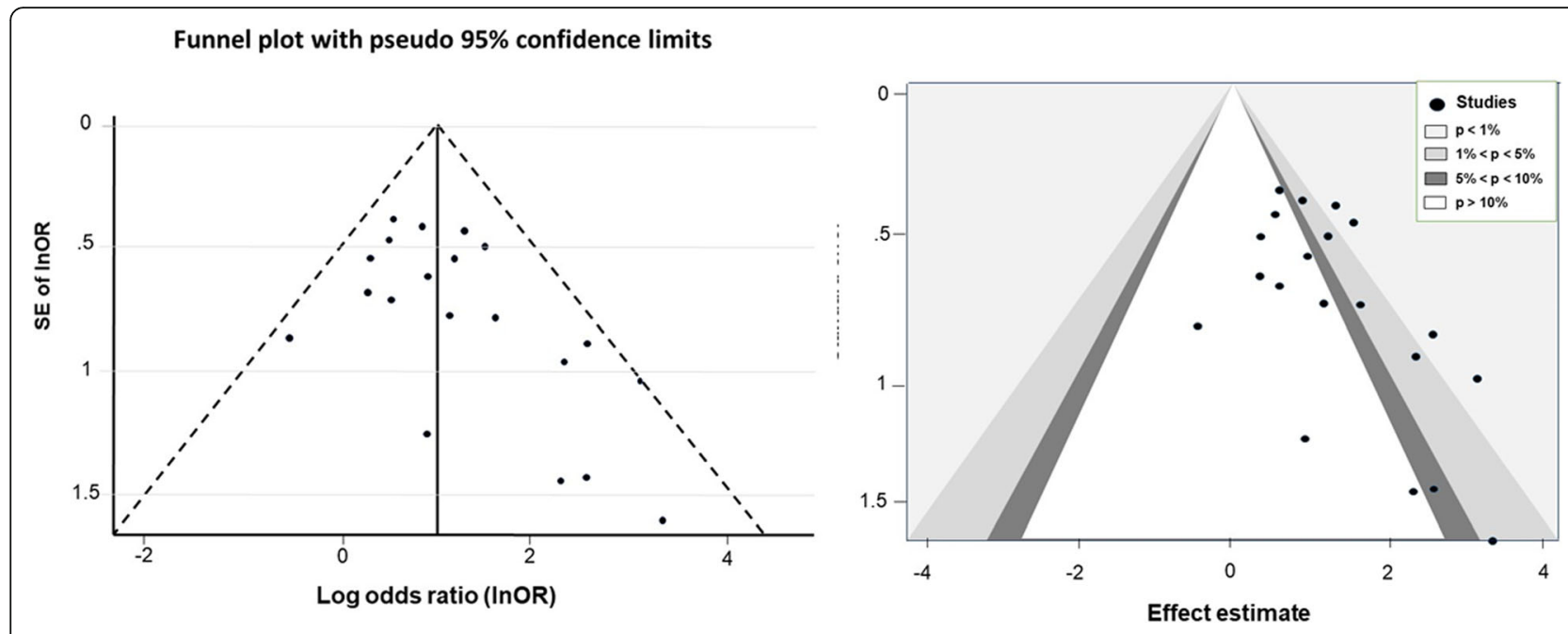

Fig. 4 Funnel plot and contour-enhanced funnel plot for less than 6 months of pre-transplant abstinence

care of psychiatric co-morbidities, and smoking cessation should be incorporated in pre-transplant selection and management periods. Careful selection of LT candidates and modifying pre-transplant risk factors of alcohol relapse has the potential to reduce alcohol relapse after LT.

\section{Abbreviations}

ALD: Alcoholic liver disease; Cl: Confidence interval; HRAR: High-Risk Alcoho Relapse; LT: Liver transplantation; OR: Odds ratio

\section{Acknowledgments}

We would like to thank the statistical team of Section for Clinical Epidemiology and Biostatistics, Faculty of Medicine, Ramathibodi Hospital for statistical analysis assistance.

\section{Authors' contributions}

LC participated in the design of the study, interpreted the data and wrote the manuscript. NY performed in the design of the study, interpreted the data and reviewed the manuscript. AT participated in the design of the study, statistical analysis, interpretation of the data and reviewed of the manuscript. AS participated in the design of the study, interpreted the data and wrote and edited the manuscript. All authors read and approved the final manuscript

\section{Funding}

This study was funded by the Gastroenterological Association of Thailand that does not have its role in the design of the study and collection, analysis, and interpretation of data and in writing the manuscript.

\section{Availability of data and materials}

Not applicable.

\section{Ethics approval and consent to participate}

Not applicable.

\section{Consent for publication}

Not applicable.

\section{Competing interests}

The authors declare that they have no competing interests.

\section{Author details}

'Division of Gastroenterology and Hepatology, Department of Medicine, Faculty of Medicine Ramathibodi Hospital, Mahidol University, 270 Rama 6 Road, Bangkok 10400, Thailand. ²Organ Transplantation Unit, Kyoto University, Kyoto, Japan. ${ }^{3}$ Section for Clinical Epidemiology and Biostatistics, Faculty of Medicine Ramathibodi Hospital, Mahidol University, Bangkok, Thailand.

Received: 8 May 2019 Accepted: 17 July 2019

Published online: 22 August 2019

\section{References}

1. Singal AK, Chaha KS, Rasheed K, Anand BS. Liver transplantation in alcoholic liver disease current status and controversies. World J Gastroenterol. 2013;19:5953-63.

2. Testino G, Burra P, Bonino F, Piani F, Sumberaz A, Peressutti R, et al. Acute alcoholic hepatitis, end stage alcoholic liver disease and liver transplantation: an Italian position statement. World J Gastroenterol. 2014; 20:14642-51.

3. Testino G, Leone S, Borro P. Alcohol and hepatocellular carcinoma: a review and a point of view. World J Gastroenterol. 2014:20:15943-54.

4. Gallegos-Orozco JF, Charlton MR. Alcoholic Liver Disease and Liver Transplantation. Clin Liver Dis. 2016;20:521-34.

5. Neuberger J, Tang H. Relapse after transplantation: European studies. Liver Transpl Surg. 1997;3:275-9.

6. Burra P, Senzolo M, Adam R, Delvart V, Karam V, Germani G, et al. Liver transplantation for alcoholic liver disease in Europe: a study from the ELTR (European Liver Transplant Registry). Am J Transplant. 2010;10:138-48.

7. Mathurin P, Moreno C, Samuel D, Dumortier J, Salleron J, Durand F, et al. Early liver transplantation for severe alcoholic hepatitis. N Engl J Med. 2011; 365:1790-800

8. Pfitzmann R, Schwenzer J, Rayes N, Seehofer D, Neuhaus R, Nussler NC. Long-term survival and predictors of relapse after orthotopic liver transplantation for alcoholic liver disease. Liver Transpl. 2007:13:197-205.

9. Schmeding M, Heidenhain C, Neuhaus R, Neuhaus P, Neumann UP. Liver transplantation for alcohol-related cirrhosis: a single centre long-term clinical and histological follow-up. Dig Dis Sci. 2011;56:236-43.

10. Cuadrado A, Fabrega E, Casafont F, Pons-Romero F. Alcohol recidivism impairs long-term patient survival after orthotopic liver transplantation for alcoholic liver disease. Liver Transpl. 2005;11:420-6.

11. Kawaguchi $Y$, Sugawara $Y$, Akamatsu N, Kaneko J, Tanaka T, Tamura S, et al. Perceptions of post-transplant recidivism in liver transplantation for alcoholic liver disease. World J Hepatol. 2014;6:812-7.

12. Bird GL, O'Grady JG, Harvey FA, Calne RY, Williams R. Liver transplantation in patients with alcoholic cirrhosis: selection criteria and rates of survival and relapse. BMJ. 1990;301:15-7. 
13. Shawcross DL, O'Grady JG. The 6-month abstinence rule in liver transplantation. Lancet. 2010;376:216-7.

14. Jauhar S, Talwalkar JA, Schneekloth T, Jowsey S, Wiesner RH, Menon KV. Analysis of factors that predict alcohol relapse following liver transplantation. Liver Transpl. 2004;10:408-11.

15. Mackie J, Groves K, Hoyle A, Garcia C, Garcia R, Gunson B, et al. Orthotopic liver transplantation for alcoholic liver disease: a retrospective analysis of survival, recidivism, and risk factors predisposing to recidivism. Liver Transpl. 2001;7:418-27.

16. Egawa H, Nishimura K, Teramukai S, Yamamoto M, Umeshita K, Furukawa H, et al. Risk factors for alcohol relapse after liver transplantation for alcoholic cirrhosis in Japan. Liver Transpl. 2014;20:298-310.

17. Immordino G, Gelli M, Ferrante R, Ferrari C, Piaggio F, Ghinolfi D, et al. Alcohol abstinence and orthotopic liver transplantation in alcoholic liver cirrhosis. Transplant Proc. 2009;41:1253-5.

18. Pageaux GP, Michel J, Coste V, Perney P, Possoz P, Perrigault PF, et al. Alcoholic cirrhosis is a good indication for liver transplantation, even for cases of recidivism. Gut. 1999;45:421-6.

19. Park YH, Hwang S, Ahn CS, Kim KH, Moon DB, Ha TY, et al. Living donor liver transplantation for patients with alcoholic liver disease. Korean J Hepatobiliary Pancreat Surg. 2013;17:14-20.

20. Bjornsson E, Olsson J, Rydell A, Fredriksson K, Eriksson C, Sjoberg C, et al. Long-term follow-up of patients with alcoholic liver disease after liver transplantation in Sweden: impact of structured management on recidivism. Scand J Gastroenterol. 2005:40:206-16.

21. Platz KP, Mueller AR, Spree E, Schumacher G, Nussler NC, Rayes N, et al. Liver transplantation for alcoholic cirrhosis. Transpl Int. 2000; 13(Suppl 1):S127-30

22. Egawa H, Ueda Y, Kawagishi N, Yagi T, Kimura H, Ichida T. Significance of pretransplant abstinence on harmful alcohol relapse after liver transplantation for alcoholic cirrhosis in Japan. Hepatol Res. 2014;44:E428-36.

23. Gedaly R, McHugh PP, Johnston TD, Jeon H, Koch A, Clifford TM, et al. Predictors of relapse to alcohol and illicit drugs after liver transplantation for alcoholic liver disease. Transplantation. 2008;86:1090-5.

24. Foster PF, Fabrega F, Karademir S, Sankary HN, Mital D, Williams JW. Prediction of abstinence from ethanol in alcoholic recipients following liver transplantation. Hepatology. 1997;25:1469-77.

25. Dew MA, DiMartini AF, Steel J, De Vito DA, Myaskovsky L, Unruh M, et al. Meta-analysis of risk for relapse to substance use after transplantation of the liver or other solid organs. Liver Transpl. 2008;14:159-72.

26. Tandon P, Goodman K, Ma MM, Wong WW, Mason AL, Meeberg G, et al. A shorter duration of pre-transplant abstinence predicts problem drinking after liver transplantation. Am J Gastroenterol. 2009;104:1700-6.

27. Duvoux C, Villamil F, Renner EL, Grazi GL, Firpi RJ, Pageaux G, et al. Sustained virological response to antiviral therapy in a randomized trial of cyclosporine versus tacrolimus in liver transplant patients with recurrent hepatitis C infection. Ann Transplant. 2015;20:25-35.

28. Zhou M, Wagner LM, Diflo T, Naegle M. Implementation of the High-Risk Alcoholism Relapse Scale in a Liver Transplant Clinic. Gastroenterol Nurs. 2015;38:447-54.

29. De Gottardi A, Spahr L, Gelez P, Morard I, Mentha G, Guillaud O, et al. A simple score for predicting alcohol relapse after liver transplantation: results from 387 patients over 15 years. Arch Intern Med. 2007;167:1183-8.

30. Starzl TE, Van Thiel D, Tzakis AG, Iwatsuki S, Todo S, Marsh JW, et al. Orthotopic liver transplantation for alcoholic cirrhosis. JAMA. 1988;260:2542-4.

31. Kumar S, Stauber RE, Gavaler JS, Basista MH, Dindzans VJ, Schade RR, et al. Orthotopic liver transplantation for alcoholic liver disease. Hepatology. 1990;11:159-64.

32. Doffoel M. Can the risk of recurrent alcohol abuse after liver transplantation for alcoholic cirrhosis be predicted? Gastroenterol Clin Biol. 2004;28:843-4.

33. Knechtle SJ, Fleming MF, Barry KL, Steen D, Pirsch JD, Hafez GR, et al. Liver transplantation for alcoholic liver disease. Surgery. 1992;112:694-701 discussion 701-693.

34. Lucey MR, Beresford TP. Alcoholic liver disease: to transplant or not to transplant? Alcohol Alcohol. 1992;27:103-8.

35. Berlakovich GA, Steininger R, Herbst F, Barlan M, Mittlbock M, Muhlbacher F. Efficacy of liver transplantation for alcoholic cirrhosis with respect to recidivism and compliance. Transplantation. 1994:58:560-5.

36. Howard L, Fahy T. Liver transplantation for alcoholic liver disease. Br J Psychiatry. 1997;171:497-500.

37. Osorio RW, Ascher NL, Avery M, Bacchetti P, Roberts JP, Lake JR. Predicting recidivism after orthotopic liver transplantation for alcoholic liver disease. Hepatology. 1994;20:105-10.
38. Gerhardt TC, Goldstein RM, Urschel HC, Tripp LE, Levy MF, Husberg BS, et al. Alcohol use following liver transplantation for alcoholic cirrhosis. Transplantation. 1996;62:1060-3.

39. Tringali RA, Trzepacz PT, DiMartini A, Dew MA. Assessment and follow-up of alcohol-dependent liver transplantation patients. A clinical cohort. Gen Hosp Psychiatry. 1996;18:70s-7s.

40. Tripp LE, Clemons JR, Goldstein RR, McCarthy Stewart L. Drinking patterns in liver transplant recipients. Psychosomatics. 1996;37:249-53.

41. Zibari GB, Edwin D, Wall L, Diehl A, Fair J, Burdick J, et al. Liver transplantation for alcoholic liver disease. Clin Transplant. 1996;10:676-9.

42. Anand AC, Ferraz-Neto BH, Nightingale P, Mirza DF, White AC, McMaster P, et al. Liver transplantation for alcoholic liver disease: evaluation of a selection protocol. Hepatology. 1997;25:1478-84.

43. Coffman KL, Hoffman A, Sher L, Rojter S, Vierling J, Makowka L. Treatment of the postoperative alcoholic liver transplant recipient with other addictions. Liver Transpl Surg. 1997;3:322-7.

44. Everson G, Bharadhwaj G, House R, Talamantes M, Bilir B, Shrestha R, et al. Long-term follow-up of patients with alcoholic liver disease who underwent hepatic transplantation. Liver Transpl Surg. 1997;3:263-74.

45. Lucey MR, Carr K, Beresford TP, Fisher LR, Shieck V, Brown KA, et al. Alcohol use after liver transplantation in alcoholics: a clinical cohort follow-up study. Hepatology. 1997;25:1223-7.

46. Reeck UH, Egerer G, Otto G, Hoffmann W, Arnold JC. Theilmann L et al: [Rehabilitation of patients with alcoholic liver cirrhosis after orthotopic liver transplantation: a 7-year follow-up]. Rehabilitation (Stuttg). 1997;36:116-20.

47. Shakil AO, Pinna A, Demetris J, Lee RG, Fung JJ, Rakela J. Survival and quality of life after liver transplantation for acute alcoholic hepatitis. Liver Transpl Surg. 1997:3:240-4.

48. Stefanini GF, Biselli M, Grazi GL, lovine E, Moscatello MR, Marsigli L, et al. Orthotopic liver transplantation for alcoholic liver disease: rates of survival, complications and relapse. Hepatogastroenterology. 1997;44:1356-9.

49. DiMartini A, Jain A, Irish W. Fitzgerald MG, Fung J. Outcome of liver transplantation in critically ill patients with alcoholic cirrhosis: survival according to medical variables and sobriety. Transplantation. 1998;66:298-302.

50. Fabrega E, Crespo J, Casafont F. De las Heras G, de la Pena J, Pons-Romero F: Alcoholic recidivism after liver transplantation for alcoholic cirrhosis. J Clin Gastroenterol. 1998;26:204-6.

51. Heinemann A, Sterneck M, Kuhlencordt R, Rogiers X, Schulz KH, Queen B, et al. Carbohydrate-deficient transferrin: diagnostic efficiency among patients with end-stage liver disease before and after liver transplantation. Alcohol Clin Exp Res. 1998;22:1806-12.

52. Tang H, Boulton R, Gunson B, Hubscher S, Neuberger J. Patterns of alcohol consumption after liver transplantation. Gut. 1998:43:140-5.

53. Conjeevaram HS, Hart J, Lissoos TW, Schiano TD, Dasgupta K, Befeler AS, et al. Rapidly progressive liver injury and fatal alcoholic hepatitis occurring after liver transplantation in alcoholic patients. Transplantation. 1999;67:1562-8.

54. Gledhill J, Burroughs A, Rolles K, Davidson B, Blizard B, Lloyd G. Psychiatric and social outcome following liver transplantation for alcoholic liver disease: a controlled study. J Psychosom Res. 1999:46:359-68.

55. Newton SE. Recidivism and return to work posttransplant. Recipients with substance abuse histories. J Subst Abuse Treat. 1999;17:103-8.

56. Romano DR, Jimenez C, Rodriguez F, Loinaz C, Colina F, Urena MA, et al. Orthotopic liver transplantation in alcoholic liver cirrhosis. Transplant Proc. 1999;31:2491-3.

57. Abosh D, Rosser B, Kaita K, Bazylewski R, Minuk G. Outcomes following liver transplantation for patients with alcohol- versus nonalcohol-induced liver disease. Can J Gastroenterol. 2000;14:851-5.

58. Berlakovich GA, Langer F, Freundorfer E, Windhager T, Rockenschaub S, Sporn E, et al. General compliance after liver transplantation for alcoholic cirrhosis. Transpl Int. 2000;13:129-35.

59. Burra P, Mioni D, Cillo U, Fagiuoli S, Senzolo M, Naccarato R, et al. Longterm medical and psycho-social evaluation of patients undergoing orthotopic liver transplantation for alcoholic liver disease. Transpl Int. 2000; 13(Suppl 1):S174-8.

60. DiMartini A, Magill J, Fitzgerald MG, Jain A, Irish W, Khera G, et al. Use of a high-risk alcohol relapse scale in evaluating liver transplant candidates. Alcohol Clin Exp Res. 2000;24:1198-201.

61. Jain A, DiMartini A, Kashyap R, Youk A, Rohal S, Fung J. Long-term follow-up after liver transplantation for alcoholic liver disease under tacrolimus. Transplantation. 2000;70:1335-42.

62. Pereira SP, Howard LM, Muiesan P, Rela M, Heaton N, Williams R. Quality of life after liver transplantation for alcoholic liver disease. Liver Transpl. 2000;6:762-8. 
63. Bellamy CO, DiMartini AM, Ruppert K, Jain A, Dodson F, Torbenson M, et al. Liver transplantation for alcoholic cirrhosis: long term follow-up and impact of disease recurrence. Transplantation. 2001;72:619-26.

64. DiMartini A, Day N, Dew MA, Lane T, Fitzgerald MG, Magill J, et al. Alcohol use following liver transplantation: a comparison of follow-up methods. Psychosomatics. 2001;42:55-62.

65. Gish RG, Lee A, Brooks L, Leung J, Lau JY, Moore DH 2nd. Long-term followup of patients diagnosed with alcohol dependence or alcohol abuse who were evaluated for liver transplantation. Liver Transpl. 2001;7:581-7.

66. Karman JF, Sileri P, Kamuda D, Cicalese L, Rastellini C, Wiley TE, et al. Risk factors for failure to meet listing requirements in liver transplant candidates with alcoholic cirrhosis. Transplantation. 2001;71:1210-3.

67. Tome S, Martinez-Rey C, Gonzalez-Quintela A, Gude F, Brage A, Otero E, et al. Influence of superimposed alcoholic hepatitis on the outcome of liver transplantation for end-stage alcoholic liver disease. J Hepatol. 2002;36:793-8.

68. Berlakovich GA, Soliman T, Freundorfer E, Windhager T, Bodingbauer M, Wamser $P$, et al. Pretransplant screening of sobriety with carbohydratedeficient transferrin in patients suffering from alcoholic cirrhosis. Transpl Int. 2004;17:617-21.

69. Miguet M, Monnet E, Vanlemmens C, Gache P, Messner M, Hruskovsky S, et al. Predictive factors of alcohol relapse after orthotopic liver transplantation for alcoholic liver disease. Gastroenterol Clin Biol. 2004;28:845-51.

70. DiMartini A, Day N, Dew MA, Javed L, Fitzgerald MG, Jain A, et al. Alcohol consumption patterns and predictors of use following liver transplantation for alcoholic liver disease. Liver Transpl. 2006;12:813-20.

71. Hwang S, Lee SG, Kim KK, Kim KH, Ahn CS, Moon DB, et al. Efficacy of 6-month pretransplant abstinence for patients with alcoholic liver disease undergoing living donor liver transplantation. Transplant Proc. 2006:38:2937-40.

72. Kelly M, Chick J, Gribble R, Gleeson M, Holton M, Winstanley J, et al. Predictors of relapse to harmful alcohol after orthotopic liver transplantation. Alcohol Alcohol. 2006;41:278-83.

73. Dumortier J, Guillaud O, Adham M, Boucaud C, Delafosse B, Bouffard Y, et al. Negative impact of de novo malignancies rather than alcohol relapse on survival after liver transplantation for alcoholic cirrhosis: a retrospective analysis of 305 patients in a single center. Am J Gastroenterol. 2007;102:1032-41.

74. Newton SE. Alcohol relapse and its relationship to the lived experience of adult liver transplant recipients. Gastroenterol Nurs. 2007;30:37-42.

75. Nickels M, Jain A, Sharma R, Orloff M, Tsoulfas G, Kashyap R, et al. Polysubstance abuse in liver transplant patients and its impact on survival outcome. Exp Clin Transplant. 2007;5:680-5.

76. Vieira A, Rolim EG, Capua A Jr, Szutan LA. Relapse of alcohol consumption in liver transplant candidates. Risk factor analysis. Arq Gastroenterol. 2007:44:205-9.

77. Wells JT, Said A, Agni R, Tome S, Hughes S, Dureja P, et al. The impact of acute alcoholic hepatitis in the explanted recipient liver on outcome after liver transplantation. Liver Transpl. 2007;13:1728-35

78. Biselli M, Gramenzi A, Del Gaudio M, Ravaioli M, Vitale G, Gitto S, et al. Long term follow-up and outcome of liver transplantation for alcoholic liver disease: a single center case-control study. J Clin Gastroenterol. 2010;44:52-7.

79. Chen GH, Yang Y, Lu MQ, Cai CJ, Zhang Q, Zhang YC, et al. Liver transplantation for end-stage alcoholic liver disease: a single-center experience from mainland China. Alcohol. 2010;44:217-21.

80. DiMartini A, Dew MA, Day N, Fitzgerald MG, Jones BL, deVera ME, et al. Trajectories of alcohol consumption following liver transplantation. Am J Transplant. 2010;10:2305-12.

81. Karim Z, Intaraprasong P, Scudamore CH, Erb SR, Soos JG, Cheung E, et al. Predictors of relapse to significant alcohol drinking after liver transplantation. Can J Gastroenterol. 2010;24:245-50.

82. Hartl J, Scherer MN, Loss M, Schnitzbauer A, Farkas S, Baier L, et al. Strong predictors for alcohol recidivism after liver transplantation: non-acceptance of the alcohol problem and abstinence of $<3$ months. Scand J Gastroenterol. 2011:46:1257-66.

83. Staufer $\mathrm{K}$, Andresen H, Vettorazzi E, Tobias N, Nashan B, Sterneck M. Urinary ethyl glucuronide as a novel screening tool in patients pre- and post-liver transplantation improves detection of alcohol consumption. Hepatology. 2011;54:1640-9.

84. Faure $S$, Herrero A, Jung B, Duny $Y$, Daures JP, Mura T, et al. Excessive alcohol consumption after liver transplantation impacts on long-term survival, whatever the primary indication. J Hepatol. 2012;57:306-12.
85. Addolorato G, Mirijello A, Leggio L, Ferrulli A, D'Angelo C, Vassallo G, et al. Liver transplantation in alcoholic patients: impact of an alcohol addiction unit within a liver transplant center. Alcohol Clin Exp Res. 2013;37:1601-8.

86. Deruytter E, Van Steenkiste C, Trepo E, Geerts A, Van Vlierberghe H, Berrevoet F, et al. Liver transplantation for alcoholic liver disease: a retrospective analysis of recidivism, survival and risk factors predisposing to alcohol relapse. Acta Gastroenterol Belg. 2013;76:282-90.

87. Kawaguchi Y, Sugawara Y, Yamashiki N, Kaneko J, Tamura S, Aoki T, et al. Role of 6-month abstinence rule in living donor liver transplantation for patients with alcoholic liver disease. Hepatol Res. 2013;43:1169-74.

88. Rice JP, Eickhoff J, Agni R, Ghufran A, Brahmbhatt R, Lucey MR. Abusive drinking after liver transplantation is associated with allograft loss and advanced allograft fibrosis. Liver Transpl. 2013;19:1377-86.

89. Rodrigue JR, Hanto DW, Curry MP. Substance abuse treatment and its association with relapse to alcohol use after liver transplantation. Liver Transplantation. 2013;19:1387-95

90. Grat M, Lewandowski Z, Grat K, Wronka KM, Krasnodebski M, Barski K, et al. Negative outcomes after liver transplantation in patients with alcoholic liver disease beyond the fifth post-transplant year. Clin Transplant. 2014;28:1112-20.

91. Piano S, Marchioro L, Gola E, Rosi S, Morando F, Cavallin M, et al. Assessment of alcohol consumption in liver transplant candidates and recipients: the best combination of the tools available. Liver Transpl. 2014; 20:815-22.

92. Dumortier J, Dharancy S, Cannesson A, Lassailly G, Rolland B, Pruvot FR, et al. Recurrent alcoholic cirrhosis in severe alcoholic relapse after liver transplantation: a frequent and serious complication. Am J Gastroenterol. 2015;110:1160-6 quiz 1167

93. Hasanin M, Dubay DA, McGuire BM, Schiano T, Singal AK. Liver transplantation for alcoholic hepatitis: A survey of liver transplant centers. Liver Transpl. 2015:21:1449-52.

94. Satapathy SK, Eason JD, Nair S, Dryn O, Sylvestre PB, Kocak M, et al. Recidivism in Liver Transplant Recipients With Alcoholic Liver Disease: Analysis of Demographic, Psychosocial, and Histology Features. Exp Clin Transplant. 2015:13:430-40.

95. Askgaard G, Tolstrup JS, Gerds TA, Hamberg O, Zierau L, Kjaer MS. Predictors of heavy drinking after liver transplantation for alcoholic liver disease in Denmark (1990-2013): a nationwide study with competing risks analyses. Scand J Gastroenterol. 2016;51:225-35.

96. Hajifathalian K, Humberson A, Hanouneh MA, Barnes DS, Arora Z, Zein NN, et al. Ohio solid organ transplantation consortium criteria for liver transplantation in patients with alcoholic liver disease. World J Hepatol. 2016;8:1149-54.

97. Im GY, Kim-Schluger L, Shenoy A, Schubert E, Goel A, Friedman SL, et al. Early Liver Transplantation for Severe Alcoholic Hepatitis in the United States--A Single-Center Experience. Am J Transplant. 2016;16:841-9.

98. Kollmann D, Rasoul-Rockenschaub S, Steiner I, Freundorfer E, Gyori GP, Silberhumer $\mathrm{G}$, et al. Good outcome after liver transplantation for ALD without a 6 months abstinence rule prior to transplantation including posttransplant CDT monitoring for alcohol relapse assessment - a retrospective study. Transpl Int. 2016;29:559-67.

99. Lee BP, Chen PH, Haugen C, Hernaez R, Gurakar A, Philosophe B, et al, Three-year Results of a Pilot Program in Early Liver Transplantation for Severe Alcoholic Hepatitis. Ann Surg. 2017;265:20-9.

100. Onishi Y, Kimura H, Hori T, Kishi S, Kamei H, Kurata N, et al. Risk of alcohol use relapse after liver transplantation for alcoholic liver disease. World J Gastroenterol. 2017;23:869-75.

101. Wigg AJ, Mangira D, Chen JW, Woodman RW. Outcomes and predictors of harmful relapse following liver transplantation for alcoholic liver disease in an Australian population. Intern Med J. 2017;47:656-63.

102. Anothaisintawee T, Rattanasiri S, Ingsathit A, Attia J, Thakkinstian A. Prevalence of chronic kidney disease: a systematic review and meta-analysis. Clin Nephrol. 2009;71:244-54

103. Rustad JK, Stern TA, Prabhakar M, Musselman D. Risk factors for alcohol relapse following orthotopic liver transplantation: a systematic review. Psychosomatics. 2015;56:21-35

104. Singal AK, Kodali S, Vucovich LA, Darley-Usmar V, Schiano TD. Diagnosis and Treatment of Alcoholic Hepatitis: A Systematic Review. Alcohol Clin Exp Res. 2016:40:1390-402

105. Bravata DM, Olkin I, Barnato AE, Keeffe EB, Owens DK. Employment and alcohol use after liver transplantation for alcoholic and nonalcoholic liver disease: a systematic review. Liver Transpl. 2001;7:191-203. 
106. McCallum S, Masterton G. Liver transplantation for alcoholic liver disease: a systematic review of psychosocial selection criteria. Alcohol Alcohol. 2006;41:358-63.

107. Rodrigue JR, Hanto DW, Curry MP. Substance abuse treatment and its association with relapse to alcohol use after liver transplantation. Liver Transpl. 2013;19:1387-95.

108. Im GY, Cameron AM, Lucey MR. Liver transplantation for alcoholic hepatitis. J Hepatol. 2019;70:328-34.

109. Lim J, Curry MP, Sundaram V. Risk factors and outcomes associated with alcohol relapse after liver transplantation. World J Hepatol. 2017;9:771-80.

\section{Publisher's Note}

Springer Nature remains neutral with regard to jurisdictional claims in published maps and institutional affiliations.

Ready to submit your research? Choose BMC and benefit from:

- fast, convenient online submission

- thorough peer review by experienced researchers in your field

- rapid publication on acceptance

- support for research data, including large and complex data types

- gold Open Access which fosters wider collaboration and increased citations

- maximum visibility for your research: over $100 \mathrm{M}$ website views per year

At $B M C$, research is always in progress.

Learn more biomedcentral.com/submissions 